\title{
Resektionsausmaß und Therapiekonzept bei hereditärem, nicht Polyposis-assoziiertem kolorektalem Karzinom (HNPCC) - Indexpatient: chirurgische Strategie
}

\author{
Steffen Pistorius
}

Klinik und Poliklinik für Viszeral-, Thorax- und Gefäßchirurgie, Universitätsklinikum Carl Gustav Carus, Technische Universität Dresden, Deutschland

\section{Schlüsselwörter}

Kolorektales Karzinom · HNPCC · Prophylaktische Chirurgie

\section{Zusammenfassung}

Ursache des klinisch durch die Amsterdam-Kriterien definierten HNPCC sind hochpenetrante Keimbahnmutationen in den DNAMismatchrepair(MMR)-Genen $\mathrm{MLH1}, \mathrm{MSH}$, seltener in $\mathrm{MSH} 6$ und PMS2. Mutationsträger in diesen MMR-Genen haben ein hohes kumulatives Risiko (52-92\%) für die Entwicklung kolorektaler - einschließlich syn- und metachroner - Karzinome, die sich meist in früheren Lebensjahren als bei sporadischen Fällen entwickeln. Darüber hinaus findet sich bei diesen Mutationsträgern ein deutlich erhöhtes Risiko für extrakolonische Karzinome, insbesondere des Endometriums, seltener der Ovarien, des Magens, der ableitenden Harnwege und des Dünndarms. Aus dieser Risikokonstellation erwächst die Frage nach einem spezifischen, individualisierten Therapiekonzept bei HNPCC-Patienten bzw. Mutationsträgern. Prinzipiell könnten drei Möglichkeiten des chirurgischen Vorgehens bezüglich des Kolorektums in Frage kommen: 1. prophylaktische Resektion bei gesunden Mutationsträgern 2. onkologische Resektion bei Karzinommanifestation 3. erweiterte Resektion mit zusätzlich prophylaktischer Intention bei Manifestation des ersten Kolon- oder Rektumkarzinoms. Die erste Möglichkeit kann nach kritischer Evaluation verschiedener Argumente als Option der Prävention nicht empfohlen werden. Zur Zeit kann sicherlich auch keine endgültige Empfehlung abgegeben werden, ob die zweite oder dritte Option des operativen Vorgehens favorisiert werden sollte. Die Indikation zur prophylaktischen Hysterektomie und Oophorektomie sollte nach ausführlicher genetischer, chirurgischer und gynäkologischer Beratung bei postmenopausalen Patientinnen, die die Amsterdam-Kriterien erfüllen oder Trägerinnen einer pathogenen Keimbahnmutation in einem MMR-Gen sind und bei denen dieser Eingriff mit einer anderweitig indizierten Operation kombiniert werden kann, erwogen werden. Eine exakte Prädiktion des individuellen Risikos und Erkrankungsalters auf Grundlage der Analyse von Interaktionen zwischen endogenen und exogenen, modifizierenden Faktoren ist Voraussetzung für individuelle Empfehlungen für «maßgeschneiderte» Vorsorgeprogramme oder prophylaktische chirurgische Maßnahmen.
Key Words

Colorectal cancer $\cdot$ HNPCC $\cdot$ Prophylactic surgery

\section{Summary}

Extent of Resection and Conception of Treatment in Patients with Hereditary Nonpolyposis Colorectal Cancer - Index Patient: Surgical Strategy

Hereditary nonpolyposis colorectal cancer (HNPCC), clinically defined by the Amsterdam criteria, is caused by highly penetrant germ line mutations in DNA mismatch repair (MMR) genes, mostly in $M L H 1$ and $M S H 2$, infrequently in $M S H 6$ und PMS2. Mutation carriers are at high cumulative risk (52-92\%) for developing colorectal cancer (CRC), including syn- and metachronous colorectal carcinomas, with a younger age of onset compared with sporadic CRC. In addition, there is a remarkably increased risk in these mutation carriers for extracolonic carcinomas, especially for endometrial and ovarian carcinomas but also for gastric, ureter and renal pelvis and small bowel cancer. Therefore, the question arises if an individually tailored conception of treatment should be applied to HNPCC patients and mutation carriers. On principle, there are three options of surgical approach conceivable concerning the colorectum: i) prophylactic resection in healthy mutation carriers ii) oncological resection in the case of $C R C$ iii) extended resection with an additional prophylactic intent in the case of first CRC. After critical evaluation of various arguments, the first option cannot be recommended for CRC prevention. However, a final recommendation neither for the second nor the third option of surgical approach can be given at the moment. The indication for a prophylactic hysterectomy and oophorectomy should be weighted in the following postmenopausal patients after intensive genetic, surgical and gynecologic counselling: patients fulfilling the Amsterdam II criteria or who have been identified as mutation carriers of a disease causing germ line mutation in one of the MMR genes and who have to be operated on due to another cause. A precise prediction of the individual risk and age of onset on the basis of the analysis of interactions between endogenous and exogenous modifying factors is the precondition for recommendations concerning individually tailored surveillance or prophylactic surgery.

$\begin{array}{ll}\text { KARGER } & \text { ๑ 2006 S. Karger GmbH, Freiburg } \\ \begin{array}{ll}\text { Fax +497614520714 } & \text { Accessible online at: } \\ \text { E-mail Information@Karger.de } \\ \text { www.karger.com }\end{array} & \begin{array}{l}\text { www.karger.com/cga } \\ \text { w }\end{array}\end{array}$

Dr. med. S. Pistorius

Klinik und Poliklinik für Viszeral-, Thorax- und Gefäßchirurgie

Universitätsklinikum Carl Gustav Carus, Technische Universität Dresden

Fetscherstraße 74, 01307 Dresden, Deutschland

Tel. +49 351 458-6958, Fax -4317

E-mail: steffen.pistorius@uniklinikum-dresden.de 


\section{Einleitung}

Das kolorektale Karzinom zählt zu den häufigsten Karzinomen der westlichen Welt. Zirka 5\% dieser Tumorentität sind durch familiäre Formen bedingt. Das HNPCC bildet mit knapp 3\% aller kolorektalen Karzinome die größte Gruppe der hereditären Formen $[1,2]$.

Das HNPCC-Syndrom, das autosomal dominant vererbt wird, ist klinisch durch die Amsterdam-I- bzw. -II-Kriterien definiert [3, 4]. Fanden bei der Definition der Amsterdam-I-Kriterien ausschließlich kolorektale Karzinome Berücksichtigung, so wurden bei der Definition der Amsterdam-II-Kriterien hingegen auch HNPCC-assoziierte, extrakolonische Karzinome (siehe unten) einbezogen. Amsterdam-I-Kriterien:

1. Mindestens drei Familienangehörige mit histologisch gesichertem, kolorektalem Karzinom, wobei ein Angehöriger mit den beiden anderen erstgradig verwandt sein muss; familiäre adenomatöse Polyposis (FAP) muss ausgeschlossen sein.

2. Wenigstens zwei aufeinanderfolgende Generationen betroffen.

3. Bei mindestens einem Patienten Diagnosestellung vor dem 50. Lebensjahr.

Amsterdam-II-Kriterien:

1. Mindestens drei Familienangehörige mit histologisch gesichertem kolorektalem Karzinom oder einem Karzinom des Endometriums, Dünndarms oder Urothels (ableitende Harnwege, Nierenbecken), wobei ein Angehöriger mit den beiden anderen erstgradig verwandt sein muss; FAP muss ausgeschlossen sein.

2. Mindestens zwei aufeinanderfolgende Generationen betroffen.

3. Bei mindestens einem Patienten Diagnosestellung vor dem 50. Lebensjahr.

Ursache des HNPCC-Syndroms sind hochpenetrante Keimbahnmutationen insbesondere in den DNA-Mismatchrepair(MMR)Genen MLH1, MSH2, seltener in MSH6 und PMS2 [5-10]. Mutationsträger in diesen MMR-Genen haben ein hohes kumulatives Risiko (52-92\%) für die Entwicklung kolorektaler - einschließlich syn- und metachroner - Karzinome, die sich meist in früheren Lebensjahren (4. Dekade) als bei sporadischen Fällen entwickeln [2, 11-13]. Darüber hinaus findet sich bei diesen Mutationsträgern ein deutlich erhöhtes Risiko für extrakolonische Karzinome, insbesondere des Endometriums, des Magens, der Ovarien, der ableitenden Harnwege und des Dünndarms [2,11,13-15]

\section{Chirurgie des Kolorektums bei HNPCC-Patienten und Mutationsträgern}

Prinzipiell könnten bei HNPCC-Patienten bzw. Mutationsträgern drei Möglichkeiten des chirurgischen Vorgehens bezüglich des Kolorektums in Frage kommen:

1. prophylaktische Resektion bei gesunden Mutationsträgern,

2. onkologische Resektion bei Karzinommanifestation,

3. erweiterte Resektion mit zusätzlich prophylaktischer Intention bei Karzinommanifestation. ad 1. Prophylaktische chirurgische Therapieansätze setzen neben einem hohen prädiktiven Wert der molekularen/klinischen Diagnose bezüglich des Phänotyps eine präzise Prädiktion des Tumorrisikos und der Tumorprognose voraus. Dies ist im Fall des HNPCCSyndroms nur bedingt gegeben.

Für eine prophylaktische Kolektomie vor der Entwicklung eines kolorektalen Karzinoms sprechen

- das hohe kumulative Risiko für kolorektale Karzinome (mindestens $52 \%$ für weibliche und mindestens $68 \%$ für männliche Mutationsträger) [2],

ein Risiko von bis zu 40\% für das Auftreten metachroner kolorektaler Karzinome [16, 17].

- ein Risiko von etwa 18\% für das Auftreten kolorektaler Karzinome trotz regelmäßiger Vorsorge im 3-Jahres-Intervall [18].

- das meist junge Erkrankungsalter.

Gegen eine prophylaktische Kolektomie vor der Entwicklung eines kolorektalen Karzinoms spricht jedoch Folgendes:

- Die inkomplette Penetranz; mindestens ein Drittel der Mutationsträger werden im Laufe ihres Lebens kein kolorektales Karzinom entwickeln und würden daher letztlich umsonst kolektomiert werden.

- Das verbliebene Rektum müsste weiterhin in regelmäßigen Intervallen überwacht und kontrolliert werden, da ein Rektumkarzinomrisiko von zirka 3\% alle 3 Jahre in den ersten 12 Jahren nach Kolektomie besteht [19].

- Ein erhebliches Risiko für die Entwicklung extrakolonischer Karzinome. Welches Karzinom sich in welchem Zeitraum bei welchem Mutationsträger entwickelt, bleibt nach wie vor unklar.

- Es besteht keine klare Genotyp-Phänotyp-Korrelation. Zwar konnte gezeigt werden, dass offensichtlich eine gewisse Abhängigkeit des Phänotyps (Manifestationsalter und -lokalisation der Karzinome) vom Genotyp (mutiertes MMR-Gen) besteht (extrakolonische Karzinome treten z. B. deutlich häufiger bei Patienten mit MSH2- und MSH6-Mutationen als bei solchen mit MLH1-Mutationen auf [11, 20-23]), jedoch lässt sich daraus keine Spezifizierung des individuellen Risikos bzw. Vorsorgeprogramms ableiten.

Letztlich kann nach kritischer Evaluation der aufgeführten Argumente die Option der prophylaktischen Kolektomie zur Prävention von Kolonkarzinomen nicht empfohlen werden.

ad 2. Die onkologische Resektion bei kolorektaler Karzinommanifestation gilt (zumindest in Deutschland) noch immer prinzipiell als das Standardvorgehen, insbesondere wenn die Daten und Angaben bezüglich der Familienanamnese unsicher oder inkomplett sind bzw. wenn das Ergebnis der molekularen Diagnostik unbekannt ist. Es muss jedoch bedacht werden, dass Patienten mit kolorektalem Karzinom, in deren Familie die Amsterdam-Kriterien tatsächlich erfüllt sind, das oben erwähnte, hohe Risiko für die Entwicklung metachroner kolorektaler Karzinome haben. Dies sollte in der entsprechenden klinischen Beratung und Aufklärung zur Operation durchaus Erwähnung finden.

ad 3. Die erweiterte Resektion mit zusätzlicher prophylaktischer Intention bei Erstmanifestation im Sinn der Kolektomie bei Ko- 
lonkarzinom bzw. (gegebenenfalls restaurativer) Proktokolektomie bei Rektumkarzinom stellt die dritte Option und in gewisser Weise einen Kompromiss dar. Die Durchführung einer Operation ist aufgrund des vorliegenden Karzinoms in jedem Falle indiziert. Diese Option schließt jedoch die Reduktion des Risikos für metachrone kolorektale Karzinome mit in die Konzeption ein. Andererseits wird vermutlich mindestens die Hälfte der Patienten im Laufe ihres weiteren Lebens nie ein metachrones kolorektales Karzinom entwickeln. Darüber hinaus muss erneut auf das oben erwähnte Risiko für extrakolonische Karzinome und die sehr unscharfe Phänotyp-Genotyp-Korrelation hingewiesen werden.

Zusammenfassend kann zurzeit sicher keine endgültige Empfehlung abgegeben werden, ob die zweite oder dritte Option des operativen Vorgehens favorisiert werden sollte. Die in Deutschland von den Zentren Düsseldorf und Dresden initiierte und von der Deutschen Krebshilfe geförderte «HNPCC-Studie zum Vergleich einer prophylaktisch erweiterten Chirurgie versus einer onkologischen Resektion beim Kolon- oder Rektumkarzinom» hatte die Klärung dieser offenen Frage zum Ziel. Jedoch musste die Studie leider im März 2006 aufgrund der ungenügenden Zahl randomisierter Patienten abgebrochen werden. Somit müssen in die sehr individuelle Entscheidungsfindung bezüglich der zu empfehlenden Therapie weiterhin die aktuell klinischen, anamnestischen, familienanamnestischen, molekularen und psychologischen Aspekte und Befunde der einzelnen Patienten einfließen.

\section{Prophylaktische Hysterektomie und Oophorektomie bei HNPCC-Patientinnen}

Hinsichtlich des Risikos für extrakolonische Karzinome stehen Endometriumkarzinome an der Spitze mit einem kumulativen Risiko von über $60 \%$ bezogen auf die Gesamtlebenszeit [2, 11-13, 15, 24]. Es konnte gezeigt werden, dass das Risiko für Endometriumkarzinome bei MSH2-Mutationsträgerinnen höher ist als bei MLH1-Mutationsträgerinnen [20, 22]. Darüber hinaus geht man bei Trägerinnen von MSH6-Mutationen sogar von einem kumulativen Risiko von bis zu 73\% aus [21, 23]. Die HNPCC-assoziierten Endometriumkarzinome treten mit einem Altersgipfel zwischen dem 40. und 62. Lebensjahr auf - signifikant zeitiger als in der Gesamtbevölkerung [24]. Typische pathohistologische Kriterien sind dabei, im Unterschied zu sporadischen Endometriumkarzinomen und ähnlich wie bei HNPCC-assoziierten kolorektalen Karzinomen, niedriger Differenzierungsgrad, Crohn-ähnliche lymphoide Reaktionen, lymphangioinvasives Wachstum und die hohe Zahl tumorinfiltrierender Lymphozyten [25].

Das Risiko für Ovarialkarzinome wird mit rund $12 \%$ angegeben [13, 26-28]. Diese beiden Tumorentitäten werden daher im HNPCC-Vorsorgeprogramm [29-31] berücksichtigt. Die diesbezügliche Effektivität des Vorsorgeprogramms ist jedoch weitgehend unbekannt. Bislang wurde nur über wenige Fälle präkanzeröser Läsionen berichtet, die bei klinischer gynäkologischer Untersuchung und endovaginaler Sonographie diagnostiziert wurden $[26,32]$.
Unserer Meinung nach sollte die Indikation zur prophylaktischen Hysterektomie und gegebenenfalls Oophorektomie bei folgenden Patientinnen nach ausführlicher präoperativer genetischer, chirurgischer und gynäkologischer Beratung erwogen werden [33]: Patientinnen, deren Anamnese und Familienanamnese die Amsterdam-Kriterien erfüllen oder die als Trägerinnen einer pathogenen Keimbahnmutation in den MMR-Genen MLH1, MSH2 oder MSH6 identifiziert wurden und postmenopausal sind und bei denen dieser Eingriff mit einer anderweitig indizierten Operation (z.B. wegen eines bestehenden kolorektalen Karzinoms) kombiniert werden kann.

Bei insgesamt 4 Patientinnen, die diese Voraussetzungen erfüllten, führten wir eine prophylaktische Hysterektomie und Oophorektomie durch und fanden bei 2 Patientinnen ein okkultes Endometriumkarzinom [33]. Über einen weiteren Fall eines okkulten Endometriumkarzinoms bei einer 48-jährigen HNPCC-Patientin, die prophylaktisch hysterektomiert und oophorektomiert worden war, wurde in einer US-amerikanischen Kasuistik berichtet [34]. In einer neuen, ebenfalls US-amerikanischen Studie [35] wurde eine prophylaktische Hysterektomie bei 61 und eine bilaterale Salpingo-Oophorektomie bei 47 HNPCC-Mutationsträgerinnen durchgeführt; bei keiner dieser Patientinnen fand sich ein Endometriumoder Ovarialkarzinom, im Gegensatz zu einer Vergleichsgruppe von 210 bzw. 223 Mutationsträgerinnen, bei denen diese prophylaktischen Eingriffe nicht erfolgten und von denen im gleichen Zeitraum 33\% ein Endometriumkarzinom und 5\% ein Ovarialkarzinom entwickelten. Diese Daten zeigten erstmals, dass diese prophylaktische Chirurgie eine effektive Strategie zur Prävention von Endometrium- und Ovarialkarzinomen bei HNPCC-Patientinnen darstellen könnte.

Da für dieses Vorgehen jedoch keine Daten prospektiver, randomisierter Studien vorliegen, sollte auch dieser Eingriff letztlich als individuelles Konzept intensiv mit den betroffenen Patientinnen diskutiert und interdisziplinär realisiert werden.

\section{Ausblick}

Obwohl die Ergebnisse der Studien der letzten Jahre einen deutlichen Zugewinn an Informationen über die grundlegenden molekularen und klinischen Zusammenhänge ermöglichten, bleibt eine Vielzahl von Fragen und Aspekten bezüglich der operativen Behandlung von HNPCC-Patienten weiterhin unbeantwortet. Von zentraler Bedeutung erscheint insbesondere die Frage, welche relevanten den Phänotyp modifizierenden molekularen Einzelnukleotidpolymorphismen (single nucleotid polymorphisms; SNPs) und exogenen Faktoren existieren und wie diese wirken. Für die Erarbeitung einer Genotyp-Phänotyp-Korrelation bei HNPCC-Patienten gibt es dazu jedoch bereits einige Ansätze.

Extrakolonische Karzinome treten häufiger bei Patienten mit MSH2- und MSH6-Mutationen auf als bei solchen mit MLH1-Mutationen [11, 20-23]. Bei Mutationsträgern in MSH6 scheint das Erkrankungsalter für kolorektale Karzinome höher, deren Inzi- 
denz jedoch geringer zu sein als bei Mutationsträgern in $M L H 1$ bzw. MSH2 [36, 37].

Eine finnische Studie [38] konnte in einer Subgruppe von HNPCCPatienten einen Zusammenhang zwischen dem GlutathionS-Transferase(GST)-M1- und T1-Null-Genotyp bzw. dem N-Acetyltransferase(NAT)-1*10+-Subtyp und einem frühen Erkrankungsalter nachweisen.

Obwohl frühere Studien auch einen Einfluss des NAT2-Acetylatortyps als modifizierenden Faktor in Erwägung gezogen hatten $[39,40]$, konnten wir in einer Studie des Deutschen HNPCC-Konsortiums bei 226 Patienten zeigen, dass der NAT2-Acetylatortyp weder einen Risikofaktor für die Entwicklung noch einen modifizierenden Faktor hinsichtlich des Erkrankungsalters für ein kolorektales Karzinom darstellt [41].

Obwohl sich in einer Studie [42] bei der Analyse des häufigen A/G-SNPs in Exon 4 des Cyclin-D1-Gens keine Korrelation zwischen einem bestimmten Allel und dem Erkrankungsalter zeigte, war die Präsenz des Transkripts b im Vergleich zum Transkript a mit einem niedrigeren Erkrankungsalter bei Kolonkarzinomen assoziiert.

Ein bei 109 HNPCC-Patienten analysierter SNP im ATM-Gen zeigte hingegen keinen Einfluss auf das Erkrankungsalter [43].

Demgegenüber fand sich eine Korrelation zwischen dem SNP Arg462Gln in RNASEL und dem Erkrankungsalter an einem kolorektalen Karzinom bei 251 Patienten mit pathogener Mutation in $M L H 1$ bzw. $M S H 2$, die durch die Zentren des Deutschen HNPCC-Konsortiums rekrutiert worden waren [44].
Eine weitere Korrelation zwischen dem Cytosin-Adenin(CA)-Dinukleotid-Repeat-Längenpolymorphismus im Promotor des Insulin-like-growth-factor-I(IGF-I)-Gens und dem Risiko für ein kolorektales Karzinom bzw. dem Erkrankungsalter konnte kürzlich in einer Studie gezeigt werden, in der 121 Patienten mit Mutationen in MMR-Genen untersucht wurden [45].

Schließlich wurde die diesbezügliche Relevanz des Arginin/ProlinSNP in Codon 72 des p53-Gens in mehreren Studien untersucht. Während zwei Studien, die 193 [46] bzw. 218 HNPCC-Patienten [47] umfassten, keine entsprechende Assoziation nachweisen konnten, fand sich in der Analyse von 167 Patienten des Deutschen HNPCC-Konsortiums eine signifikante Korrelation zwischen diesem Polymorphismus und dem Erkrankungsalter an einem kolorektalen Karzinom [48].

Zusammenfassend lässt sich festhalten, dass in den bisher durchgeführten Studien zur Genotyp-Phänotyp-Korrelation bei HNPCCPatienten zwar eine Reihe genetisch modifizierender Faktoren identifiziert werden konnte, eine exakte Prädiktion des individuellen Risikos und Erkrankungsalters und damit individuelle Empfehlungen bezüglich eines «maßgeschneiderten» Vorsorgeprogramms oder prophylaktischer chirurgischer Maßnahmen jedoch noch immer nicht möglich sind. Zukünftige Studien werden sich daher noch intensiver den möglichen funktionellen Interaktionen innerhalb und zwischen den endogenen und exogenen modifizierenden Faktoren zuwenden müssen.

\section{Literatur}

1 Kerber RA, Neklason DW, Samowitz WS, Burt RW: Frequency of familial colon cancer and hereditary nonpolyposis colorectal cancer (Lynch syndrome) in a large population database. Fam Cancer 2005;4:239-244.

2 Hampel H, Stephens JA, Pukkala E, Sankila R, Aaltonen LA, Mecklin JP, de la Chapelle A: Cancer risk in hereditary nonpolyposis colorectal cancer syndrome: later age of onset. Gastroenterology 2005;129:415-421.

3 Vasen HF, Mecklin JP, Khan PM, Lynch HT: The International Collaborative Group on Hereditary Non-Polyposis Colorectal Cancer (ICGHNPCC). Dis Colon Rectum 1991;34:424-425.

4 Vasen HF, Watson P, Mecklin JP, Lynch HT: New clinical criteria for hereditary nonpolyposis colorectal cancer (HNPCC, Lynch syndrome) proposed by the International Collaborative Group on HNPCC. Gastroenterology 1999;116:14531456.

5 Fishel R, Lescoe MK, Rao MR, Copeland NG, Jenkins NA, Garber J, Kane M, Kolodner R: The human mutator gene homolog MSH2 and its association with hereditary nonpolyposis colon cancer. Cell 1993;75:1027-1038.

6 Bronner CE, Baker SM, Morrison PT, Warren G, Smith LG, Lescoe MK, Kane M, Earabino C, Lipford J, Lindblom A, et al: Mutation in the DNA mismatch repair gene homologue hMLH1 is associated with hereditary non-polyposis colon cancer. Nature 1994;368:258-261.
7 Nicolaides NC, Papadopoulos N, Liu B, Wei YF, Carter KC, Ruben SM, Rosen CA, Haseltine WA, Fleischmann RD, Fraser CM, et al: Mutations of two PMS homologues in hereditary nonpolyposis colon cancer. Nature 1994;371:75-80.

8 Miyaki M, Konishi M, Tanaka K, KikuchiYanoshita R, Muraoka M, Yasuno M, Igari T, Koike M, Chiba M, Mori T: Germline mutation of MSH6 as the cause of hereditary nonpolyposis colorectal cancer. Nat Genet 1997;17:271-272.

9 Peltomaki P, Vasen H: Mutations associated with HNPCC predisposition - update of ICGHNPCC/INSiGHT mutation database. Dis Markers 2004;20:269-276.

10 Mangold E, Pagenstecher C, Friedl W, Mathiak M, Buettner R, Engel C, Loeffler M, Holinski-Feder E, Muller-Koch Y, Keller G, Schackert HK, Kruger S, Goecke T, Moeslein G, Kloor M, Gebert J, Kunstmann E, Schulmann K, Ruschoff J, Propping P: Spectrum and frequencies of mutations in MSH2 and MLH1 identified in 1,721 German families suspected of hereditary nonpolyposis colorectal cancer. Int $\mathrm{J}$ Cancer 2005;116:692-702.

11 Vasen HF, Wijnen JT, Menko FH, Kleibeuker JH, Taal BG, Griffioen G, Nagengast FM, Meijers-Heijboer EH, Bertario L, Varesco L, Bisgaard ML, Mohr J, Fodde R, Khan PM: Cancer risk in families with hereditary nonpolyposis colorectal cancer diagnosed by mutation analysis. Gastroenterology 1996;110:1020-1027.
12 Dunlop MG, Farrington SM, Carothers AD, Wyllie AH, Sharp L, Burn J, Liu B, Kinzler KW, Vogelstein B: Cancer risk associated with germline DNA mismatch repair gene mutations. Hum Mol Genet 1997;6:105-110.

13 Aarnio M, Sankila R, Pukkala E, Salovaara R, Aaltonen LA, de la Chapelle A, Peltomaki P, Mecklin JP, Jarvinen HJ: Cancer risk in mutation carriers of DNA-mismatch-repair genes. Int $\mathrm{J}$ Cancer 1999;81:214-218.

14 Sijmons RH, Kiemeney LA, Witjes JA, Vasen HF: Urinary tract cancer and hereditary nonpolyposis colorectal cancer: risks and screening options. J Urol 1998;160:466-470.

15 Watson P, Lynch HT: Cancer risk in mismatch repair gene mutation carriers. Fam Cancer 2001; 1:57-60.

16 Lynch HT: Is there a role for prophylactic subtotal colectomy among hereditary nonpolyposis colorectal cancer germline mutation carriers? Dis Colon Rectum 1996;39:109-110.

17 Vasen HF, Nagengast FM, Khan PM: Interval cancers in hereditary non-polyposis colorectal cancer (Lynch syndrome). Lancet 1995;345:11831184.

18 Jarvinen HJ, Aarnio M, Mustonen H, Aktan-Collan K, Aaltonen LA, Peltomaki P, De La Chapelle A, Mecklin JP: Controlled 15-year trial on screening for colorectal cancer in families with hereditary nonpolyposis colorectal cancer. Gastroenterology 2000;118:829-834. 
19 Rodriguez-Bigas MA, Vasen HF, Pekka-Mecklin J, Myrhoj T, Rozen P, Bertario L, Jarvinen HJ, Jass JR, Kunitomo K, Nomizu T, Driscoll DL: Rectal cancer risk in hereditary nonpolyposis colorectal cancer after abdominal colectomy. International Collaborative Group on HNPCC. Ann Surg 1997;225:202-207.

20 Lin KM, Shashidharan M, Thorson AG, Ternent CA, Blatchford GJ, Christensen MA, Watson P, Lemon SJ, Franklin B, Karr B, Lynch J, Lynch HT: Cumulative incidence of colorectal and extracolonic cancers in MLH1 and MSH2 mutation carriers of hereditary nonpolyposis colorectal cancer. J Gastrointest Surg 1998;2:67-71.

21 Wijnen J, de Leeuw W, Vasen H, van der Klift H, Moller P, Stormorken A, Meijers-Heijboer H, Lindhout D, Menko F, Vossen S, Moslein G, Tops C, Brocker-Vriends A, Wu Y, Hofstra R, Sijmons R, Cornelisse C, Morreau H, Fodde R: Familial endometrial cancer in female carriers of MSH6 germline mutations. Nat Genet 1999;23: 142-144.

22 Peltomaki P, Gao X, Mecklin JP: Genotype and phenotype in hereditary nonpolyposis colon cancer: a study of families with different vs. shared predisposing mutations. Fam Cancer 2001:1:9-15.

23 Hendriks YM, Wagner A, Morreau H, Menko F, Stormorken A, Quehenberger F, Sandkuijl L, Moller P, Genuardi M, Van Houwelingen $\mathrm{H}$, Tops C, Van Puijenbroek M, Verkuijlen P, Kenter G, Van Mil A, Meijers-Heijboer H, Tan GB, Breuning MH, Fodde R, Wijnen JT, BrockerVriends AH, Vasen H: Cancer risk in hereditary nonpolyposis colorectal cancer due to MSH6 mutations: impact on counseling and surveillance. Gastroenterology 2004;127:17-25.

24 Watson P, Vasen HF, Mecklin JP, Jarvinen H, Lynch HT: The risk of endometrial cancer in hereditary nonpolyposis colorectal cancer. Am J Med 1994;96:516-520.

25 van den Bos M, van den Hoven M, Jongejan E, van der Leij F, Michels M, Schakenraad S Aben K, Hoogerbrugge N, Ligtenberg M, van Krieken JH: More differences between HNPCC related and sporadic carcinomas from the endometrium as compared to the colon. Am J Surg Pathol 2004;28:706-711.

26 Rijcken FE, Mourits MJ, Kleibeuker JH, Hollema H, van der Zee AG: Gynecologic screening in hereditary nonpolyposis colorectal cancer. Gynecol Oncol 2003;91:74-80.

27 Watson P, Butzow R, Lynch HT, Mecklin JP, Jarvinen HJ, Vasen HF, Madlensky L, Fidalgo P, Bernstein I: The clinical features of ovarian cancer in hereditary nonpolyposis colorectal cancer. Gynecol Oncol 2001;82:223-228.

28 Brown GJ, St John DJ, Macrae FA, Aittomaki K Cancer risk in young women at risk of hereditary nonpolyposis colorectal cancer: implications for gynecologic surveillance. Gynecol Oncol 2001;80:346-349.

29 Vasen HF, Taal BG, Nagengast FM, Griffioen G, Menko FH, Kleibeuker JH, Offerhaus GJ, Meera
Khan P: Hereditary nonpolyposis colorectal cancer: results of long-term surveillance in 50 families. Eur J Cancer 1995;31A:1145-1148.

30 Burke W, Petersen G, Lynch P, Botkin J, Daly M, Garber J, Kahn MJ, McTiernan A, Offit K, Thomson E, Varricchio C: Recommendations for follow-up care of individuals with an inherited predisposition to cancer. I. Hereditary nonpolyposis colon cancer. Cancer Genetics Studies Consortium. Jama 1997:277:915-919.

31 Schmiegel W, Adler G, Fruhmorgen P, Folsch U, Graeven U, Layer P, Petrasch S, Porschen R, Pox C, Sauerbruch T, Schmoll HJ, Zeitz M: Kolorektales Karzinom: Prävention und Früherkennung in der asymptomatischen Bevölkerung Vorsorge bei Risikopatienten - Endoskopische Diagnostik, Therapie und Nachsorge von Polypen und Karzinomen.. Z Gastroenterol 2000;38: 49-75.

32 Dove-Edwin I, Boks D, Goff S, Kenter GG, Carpenter R, Vasen HF, Thomas HJ: The outcome of endometrial carcinoma surveillance by ultrasound scan in women at risk of hereditary nonpolyposis colorectal carcinoma and familial colorectal carcinoma. Cancer 2002;94:1708-1712.

33 Pistorius S, Kruger S, Hohl R, Plaschke J, Distler W, Saeger HD, Schackert HK: Occult endometrial cancer and decision making for prophylactic hysterectomy in hereditary nonpolyposis colorectal cancer patients. Gynecol Oncol 2006;102:189-194.

34 Chung L, Broaddus R, Crozier M, Luthraa R, Levenback C, Lu K: Unexpected endometrial cancer at prophylactic hysterectomy in a woman with hereditary nonpolyposis colon cancer. Obstet Gynecol 2003;102:1152-1155.

35 Schmeler KM, Lynch HT, Chen LM, Munsell MF, Soliman PT, Clark MB, Daniels MS, White KG, Boyd-Rogers SG, Conrad PG, Yang KY, Rubin MM, Sun CC, Slomovitz BM, Gershenson $\mathrm{DM}, \mathrm{Lu} \mathrm{KH}$ : Prophylactic surgery to reduce the risk of gynecologic cancers in the Lynch syndrome. N Engl J Med 2006;354:261-269.

36 Wagner A, Hendriks Y, Meijers-Heijboer EJ, de Leeuw WJ, Morreau H, Hofstra R, Tops C, Bik E, Brocker-Vriends AH, van Der Meer C, Lindhout D, Vasen HF, Breuning MH, Cornelisse CJ, van Krimpen $\mathrm{C}$, Niermeijer MF, Zwinderman AH, Wijnen J, Fodde R: A typical HNPCC owing to MSH6 germline mutations: analysis of a large Dutch pedigree. J Med Genet 2001;38:318-322.

37 Plaschke J, Engel C, Kruger S, Holinski-Feder E, Pagenstecher C, Mangold E, Moeslein G, Schulmann K, Gebert J, von Knebel Doeberitz M, Ruschoff J, Loeffler M, Schackert HK: Lower incidence of colorectal cancer and later age of disease onset in 27 families with pathogenic MSH6 germline mutations compared with families with MLH1 or MSH2 mutations: the German Hereditary Nonpolyposis Colorectal Cancer Consortium. J Clin Oncol 2004;22:4486-4494.

38 Moisio AL, Sistonen P, Mecklin JP, Jarvinen H, Peltomaki P: Genetic polymorphisms in carcino- gen metabolism and their association to hereditary nonpolyposis colon cancer. Gastroenterology 1998;115:1387-1394.

39 Heinimann K, Scott RJ, Chappuis P, Weber W, Muller H, Dobbie Z, Hutter P: N-acetyltransferase 2 influences cancer prevalence in hMLH1/ hMSH2 mutation carriers. Cancer Res 1999;59: 3038-3040.

40 Frazier ML, O’Donnell FT, Kong S, Gu X, Campos I, Luthra R, Lynch PM, Amos CI: Age-associated risk of cancer among individuals with $\mathrm{N}$ acetyltransferase 2 (NAT2) mutations and mutations in DNA mismatch repair genes. Cancer Res 2001;61:1269-1271.

41 Pistorius S, Gorgens H, Kruger S, Engel C, Mangold E, Pagenstecher C, Holinski-Feder E, Moeslein G, von Knebel Doeberitz M, Ruschoff J, Karner-Hanusch J, Saeger HD, Schackert HK, The German HNPCC-Consortium: N-acetyltransferase (NAT) 2 acetylator status and age of onset in patients with hereditary nonpolyposis colorectal cancer (HNPCC). Cancer Lett 2005; 241:150-157.

42 Bala S, Peltomaki P: CYCLIN D1 as a genetic modifier in hereditary nonpolyposis colorectal cancer. Cancer Res 2001;61:6042-6045.

43 Jones JS, Gu X, Lynch PM, Rodriguez-Bigas M, Amos CI, Frazier ML: ATM polymorphism and hereditary nonpolyposis colorectal cancer (HNPCC) age of onset (United States). Cancer Causes Control 2005;16:749-753.

44 Kruger S, Silber AS, Engel C, Gorgens H, Mangold E, Pagenstecher C, Holinski-Feder E, von Knebel Doeberitz M, Moeslein G, Dietmaier W, Stemmler S, Friedl W, Ruschoff J, Schackert HK Arg462Gln sequence variation in the prostatecancer-susceptibility gene RNASEL and age of onset of hereditary non-polyposis colorectal cancer: a case-control study. Lancet Oncol 2005;6: 566-572.

45 Zecevic M, Amos CI, Gu X, Campos IM, Jones JS, Lynch PM, Rodriguez-Bigas MA, Frazier ML: IGF1 gene polymorphism and risk for hereditary nonpolyposis colorectal cancer. J Natl Cancer Inst 2006;98:139-143.

46 Sotamaa K, Liyanarachchi S, Mecklin JP, Jarvinen H, Aaltonen LA, Peltomaki P, de la Chapelle A: p53 codon 72 and MDM2 SNP309 polymorphisms and age of colorectal cancer onset in Lynch syndrome. Clin Cancer Res 2005;11:6840 6844.

47 Talseth BA, Meldrum C, Suchy J, Kurzawski G, Lubinski J, Scott RJ: Age of diagnosis of colorectal cancer in HNPCC patients is more complex than that predicted by $\mathrm{R} 72 \mathrm{P}$ polymorphism in TP53. Int J Cancer 2006;118:2479-2484.

48 Kruger S, Bier A, Engel C, Mangold E, Pagenstecher C, von Knebel Doeberitz M, Holinski-Feder E, Moeslein G, Schulmann K, Plaschke J, Ruschoff J, Schackert HK: The p53 codon 72 variation is associated with the age of onset of hereditary non-polyposis colorectal cancer (HNPCC). J Med Genet 2005;42:769-773. 